\title{
Consensus recommendations for MS cortical lesion scoring using double inversion recovery MRI
}

J.J.G. Geurts, PhD

S.D. Roosendaal, MD

M. Calabrese, MD

O. Ciccarelli, PhD

F. Agosta, MD

D.T. Chard, PhD

A. Gass, MD

E. Huerga, $\mathrm{PhD}$

B. Moraal, MD

D. Pareto, PhD

M.A. Rocca, MD

M.P. Wattjes, MD

T.A. Yousry, MD

B.M.J. Uitdehaag, MD

F. Barkhof, MD

On behalf of the MAGNIMS Study Group

Address correspondence and reprint requests to Dr. Jeroen J.G. Geurts, VU University Medical Center, Department of Anatomy \& Neuroscience, MF G-116, van der Boechorststraat 7 , 1081 BT Amsterdam, the Netherlands j.geurts@vumc.nl

Editorial, page 412

\section{ABSTRACT}

Background: Different double inversion recovery (DIR) sequences are currently used in multiple sclerosis (MS) research centers to visualize cortical lesions, making it difficult to compare published data. This study aimed to formulate consensus recommendations for scoring cortical lesions in patients with MS, using DIR images acquired in 6 European centers according to local protocols.

Methods: Consensus recommendations were formulated and tested in a multinational meeting.

Results: Cortical lesions were defined as focal abnormalities on DIR, hyperintense compared to adjacent normal-appearing gray matter, and were not scored unless $\geq 3$ pixels in size, based on at least $1.0 \mathrm{~mm}^{2}$ in-plane resolution. Besides these 2 obligatory criteria, additional, supportive recommendations concerned a priori artifact definition on DIR, use of additional MRI contrasts to verify suspected lesions, and a constant level of displayed image contrast. Robustness of the recommendations was tested in a small dataset of available, heterogeneous DIR images, provided by the different participating centers. An overall moderate agreement was reached when using the proposed recommendations: more than half of the readers agreed on slightly more than half (54\%) of the cortical lesions scored, whereas complete agreement was reached in $19.4 \%$ of the lesions (usually larger, mixed white matter/gray matter lesions).

Conclusions: Although not designed as a formal interobserver study, the current study suggests that comparing available literature data on cortical lesions may be problematic, and increased consistency in acquisition protocols may improve scoring agreement. Sensitivity and specificity of the proposed recommendations should now be studied in a more formal, prospective, multicenter setting using similar DIR protocols. Neurology ${ }^{\circledR} 2011 ; 76: 418-424$

\section{GLOSSARY}

DIR = double inversion recovery; EDSS = Expanded Disability Status Scale; GM = gray matter; $\mathbf{M S}=$ multiple sclerosis; WM $=$ white matter.

Histopathology studies have shown that demyelination of the gray matter (GM) is extensive in multiple sclerosis (MS). ${ }^{1-4}$ However, visualization of cortical lesions in MS with standard MRI techniques remains difficult. ${ }^{5}$ More advanced MRI techniques, like double inversion recovery (DIR), enabled substantial improvement of cortical lesion detection, ${ }^{6-8}$ and showed that cortical lesions are associated with clinicocognitive impairment. ${ }^{9-11}$

In healthy brain, DIR visualizes GM, while suppressing the signals from white matter (WM) and CSF. ${ }^{6,12}$ Unfortunately, this double suppression scheme creates an image with low signalto-noise, which, combined with the influence of a nonuniform magnetic field in the cortex and

e-Pub ahead of print on January 5, 2011, at www.neurology.org.

From the VU University Medical Center, Departments of Radiology (S.D.R., B.M., M.P.W., F.B.), Anatomy \& Neuroscience, Division of Clinical Neuroscience (J.J.G.G.), and Department of Neurology (B.M.J.U.), Amsterdam, the Netherlands; Department of Neurosciences (M.C.), University Hospital of Padova, Multiple Sclerosis Center of Veneto Region, Padova, Italy; Departments of Brain Repair and Rehabilitation (O.C., T.A.Y.) and Neuroinflammation (D.T.C.), Institute of Neurology, University College London, London, UK; Neuroimaging Research Unit (F.A., M.A.R.), Institute of Experimental Neurology, Division of Neuroscience, Scientific Institute and University Hospital San Raffaele, Milan, Italy; Department of Neurology (A.G.), University of Basel, Basel, Switzerland; and Magnetic Resonance Unit (E.H., D.P.), Department of Radiology, Hospital Vall d'Hebron, Barcelona, Spain.

Study funding: The MS Center Amsterdam is supported by the Dutch MS Research Foundation (05-358c). The NMR Research Unit of the Department of Neuroinflammation is supported by the MS Society Great Britain and Northern Ireland, and Department of Health's NIHR Comprehensive Biomedical Research Centre at UCLH.

Disclosure: Author disclosures are provided at the end of the article. 
flow artifacts, has sparked debate as to whether all signal hyperintensities on DIR should be considered actual cortical lesions. Subsequently, high-field ${ }^{13-15}$ and (combinations of) T1-based and phase-sensitive inversion-recovery techniques ${ }^{16-19}$ were shown to improve visualization of cortical lesions. ${ }^{19}$ A recent study using advanced multicontrast 3-dimensional imaging confirmed the potential of DIR imaging, by showing that DIR permits the highest detection of cortical lesions when compared with other sequences. ${ }^{20}$

Postmortem verification of cortical hyperintensities on DIR is ongoing, but is hampered by several challenges posed by optimization of DIR for postmortem tissue imaging. Therefore, a true gold standard is currently unavailable. With this in mind, a multinational meeting was organized to reach consensus and propose recommendations for scoring cortical MS lesions on available in vivo DIR images. The results of this consensus meeting, and the first application of the recommendations to a DIR dataset, are presented here.

METHODS Participating centers. Twelve researchers with various degrees of experience in identifying MS lesions on conventional scans, from 6 specialized European MS centers (University College of London, UK; University Hospital Basel, Switzerland; University Hospital Vall d'Hebron, Barcelona, Spain; University Hospital San Raffaele, Milan, Italy; Multiple Sclerosis Center of Veneto Region, Padova, Italy; and VU University Medical Center, Amsterdam, the Netherlands) convened for a 2-day consensus meeting in Amsterdam. Before the meeting, representative DIR images from healthy controls and patients with MS were acquired in the different centers according to their local protocols, and were discussed digitally by the researchers, so that they had the opportunity to become acquainted with the different sequences before the meeting.

Collection of MRI. Prior to the consensus meeting, each center was asked to send to the meeting organizers whole-brain DIR scans of 3 patients with MS and one healthy control. This resulted in imaging data from a total of 18 patients with MS (mean age: 42 years \pm 9 years; 10 women; mean disease duration: $129 \pm 103$ months; median Expanded Disability Status Scale (EDSS) score: 3.0 [range $1.0-6.5$ ]) and 6 healthy controls (mean age: $35 \pm 6$ years; 4 women). Since these DIR images were acquired for previous studies using local protocols, the final imaging dataset was heterogeneous. In particular, $1.5 \mathrm{~T}$ scanners were used in 3 centers, $3 \mathrm{~T}$ scanners in 2 centers, and both $1.5 \mathrm{~T}$ and $3 \mathrm{~T}$ scanners in one center. In addition, 4 centers acquired 3-dimensional images, while 2 centers acquired 2-dimensional images. Sequences featured different slice thicknesses (mean 1.4 $\mathrm{mm}$; range $1.3-3.0 \mathrm{~mm}$ ), spatial resolution (mean $1.21 \mathrm{~mm}^{2}$; range $0.98-1.3 \mathrm{~mm}^{2}$ ), and number of slices (mean 124; range 44-256). For an illustration of the collected DIR images from the different centers, and differences in parameters, see the table and figure 1 .

Definition of recommendations. The consensus meeting was conducted in 2 parts. During the first day, the 12 meeting participants discussed the definition of formal criteria for international cortical lesion scoring, based on DIR imaging. The discussion was supported by 2 practice sessions, during which readers applied interim recommendations to a test set of DIR images (of both patients and healthy controls, selected from existing databases from Padova, Amsterdam, and Milan). This allowed them to share experiences concerning the feasibility of interim recommendations, upon which suggestions for modification and refinement were discussed. At the end of the first day, final scoring recommendations were formulated and agreed upon. It was decided to score cortical lesions, which had to be entirely or partly located in the cortical GM, but that subpial demyelination $^{2}$ and lesions located in WM directly adjacent to the cortex (i.e., not entering the cortical GM) would not be scored.

Application of the recommendations using the collected data. On the second day of the meeting and after a brief recapitulation of the defined scoring recommendations, 5 teams, consisting of 2 or 3 readers each, scored the collected DIR images (axial representation) of the 18 patients with MS and 6 healthy controls (described above). The use of images from hu-

\begin{tabular}{|c|c|c|c|c|c|c|c|}
\hline DIR sequenc & e parameter & from the dit & ferent sites & & & & \\
\hline Sequence parameters & London & Basel & Barcelona & Milan & Milan & Padova & Amsterdam \\
\hline Field strength & $3.0 \mathrm{~T}$ & $1.5 \mathrm{~T}$ & $1.5 \mathrm{~T}$ & $1.5 \mathrm{~T}$ & $3.0 \mathrm{~T}$ & $1.5 \mathrm{~T}$ & $1.5 \mathrm{~T}$ \\
\hline TR, ms & 10,000 & 7,500 & 2,350 & 11,000 & 11,000 & 15,631 & 6,500 \\
\hline$T E, m s$ & 83 & 311 & 356 & 25 & 25 & 25 & 355 \\
\hline $\mathrm{Tl}_{1,2}, \mathrm{~ms}$ & $510,3,000$ & $500,3,000$ & $350,2,350$ & $325,3,400$ & $325,3,400$ & $325,3,400$ & $350,2,350$ \\
\hline Slice thickness, mm & 1.3 & 1.5 & 1.3 & 3.0 & 5.0 & 3.0 & 1.2 \\
\hline No. of slices & 120 & 128 & 120 & 44 & 24 & 50 & 256 \\
\hline $\begin{array}{l}\text { In-plane resolution, } \\
\mathrm{mm} \times \mathrm{mm}\end{array}$ & $1.2 \times 1.2$ & $1.3 \times 1.3$ & $1.3 \times 1.3$ & $1.0 \times 1.1$ & $0.9 \times 1.2$ & $1.9 \times 0.8$ & $1.2 \times 1.3$ \\
\hline $2 D / 3 D$ & $3 D$ & $3 D$ & $3 D$ & $2 \mathrm{D}$ & $2 \mathrm{D}$ & $2 \mathrm{D}$ & $3 D$ \\
\hline Acquisition time, min:sec & $15: 00$ & 08:02 & 09:45 & $06: 03$ & $08: 00$ & 05:31 & 09:50 \\
\hline
\end{tabular}

Abbreviations: $\mathrm{DIR}=$ double inversion recovery; $\mathrm{TE}=$ echo time; $\mathrm{TI}=$ inversion time; $\mathrm{TR}=$ repetition time. 

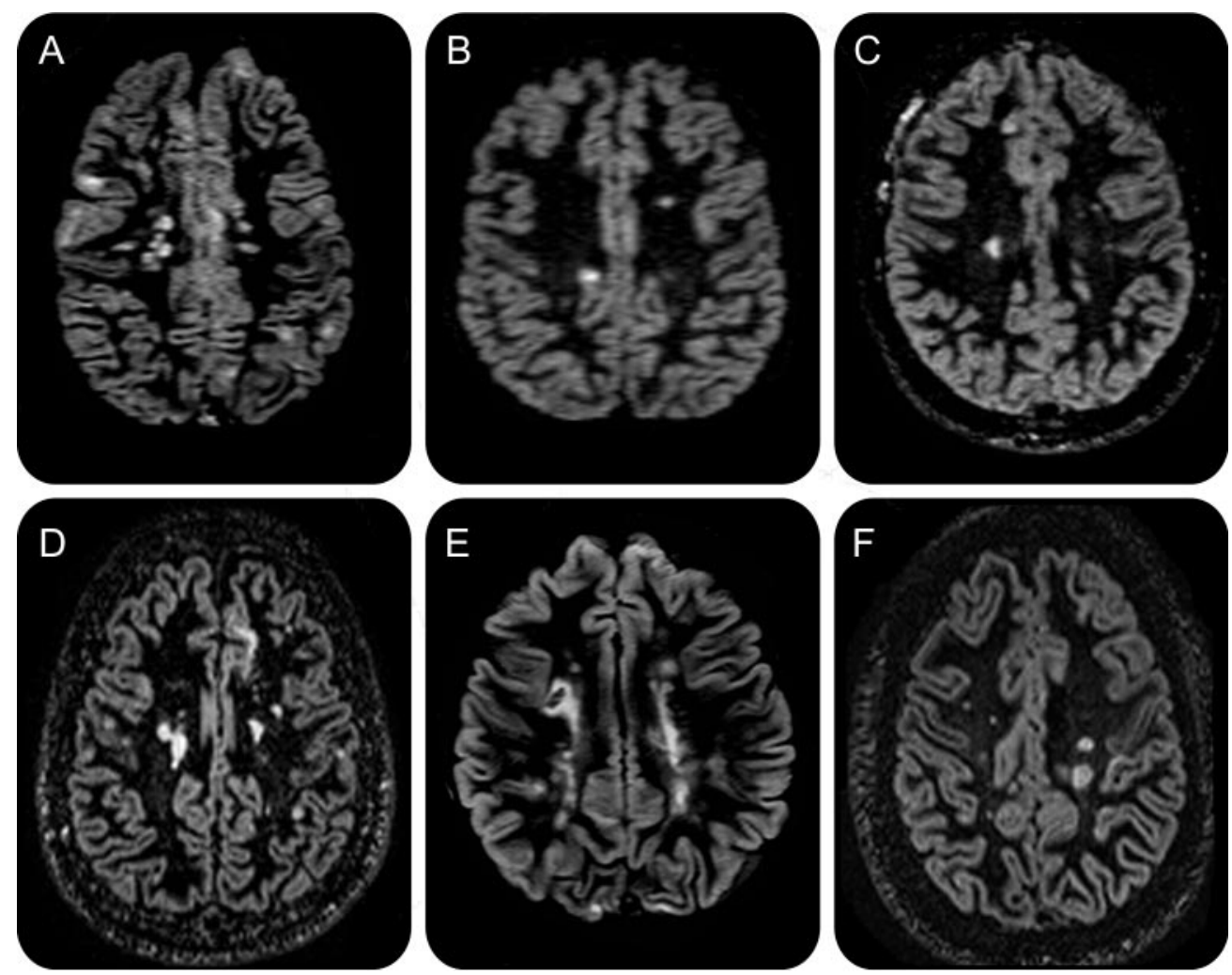

(A) DIR from Padua (1.5 T), (B) Barcelona (1.5 T), (C) Amsterdam (1.5 T), (D) Basel (1.5 T), (E) Milan (3.0 T), and (F) London (3.0 T).

man subjects for this study was approved by the local Institutional Review Board, and written informed consent was obtained from all participants. Data were fully anonymized, i.e., no patient, site, or sequence information was visible on the images. Teams of readers, instead of single raters, were chosen to stimulate discussion between participants about the application of the newly proposed scoring recommendations. DIR hyperintensities were marked as lesions if consensus was reached between the members of a given team.

All scorings were analyzed after the meeting and a comparison of lesion ratings between teams was made. Agreement between rating teams on the level of individual lesions was assessed, by noting whether $\geq 3, \geq 4$, or all 5 teams had scored the same lesion. Similarly, agreement was investigated on the patient level, i.e., the total cortical lesion number per patient, by calculating a coefficient of variation.

RESULTS Recommendations for cortical lesion scoring in patients with MS. The meeting resulted in the definition of a set of to-the-point recommendations for cortical lesion scoring.

It was deemed obligatory that:

1. Cortical lesions are hyperintense on DIR images, compared to surrounding normal-appearing GM.

2. Moreover, cortical hyperintensities should occupy at least 3 pixels (based on minimally $1 \mathrm{~mm}^{2}$ in-plane resolution).

Furthermore, the following points were suggested as supportive recommendations:
1. Readers should become acquainted with common artifacts (i.e., unwanted changes in signal intensity) on DIR. Several areas in the brain seem to be especially artifact-prone on DIR (for an illustration of common [sites of] artifacts on DIR, see figure 2). Readers should be familiarized with these artifacts using healthy control DIR images acquired with the same imaging parameters as those used for the MS patient scans to be scored.

2. Near-constant image contrast settings should be maintained while scoring, to avoid confusion resulting from inherent inhomogeneous signal abnormalities (subtle and diffuse hyperintensities) in the cortex.

3. Multiple slices may need to be viewed when determining whether or not a DIR signal hyperintensity should be scored as a cortical lesion; in this way, lesions can be distinguished from (round, traceable) cortical vessels (see figure 2). A slightly irregular shape (as opposed to neatly rounded vessels) of a hyperintensity may also be a supporting feature of demyelination.

4. Other MRI contrasts, notably T1-based and T2 or fluid-attenuated inversion recovery images (preferably 3-dimensional isotropic volumes to allow reformatting and optimal matching with 
Figure 2 Common (sites of) artifacts on double inversion recovery (DIR) images (healthy control subject)
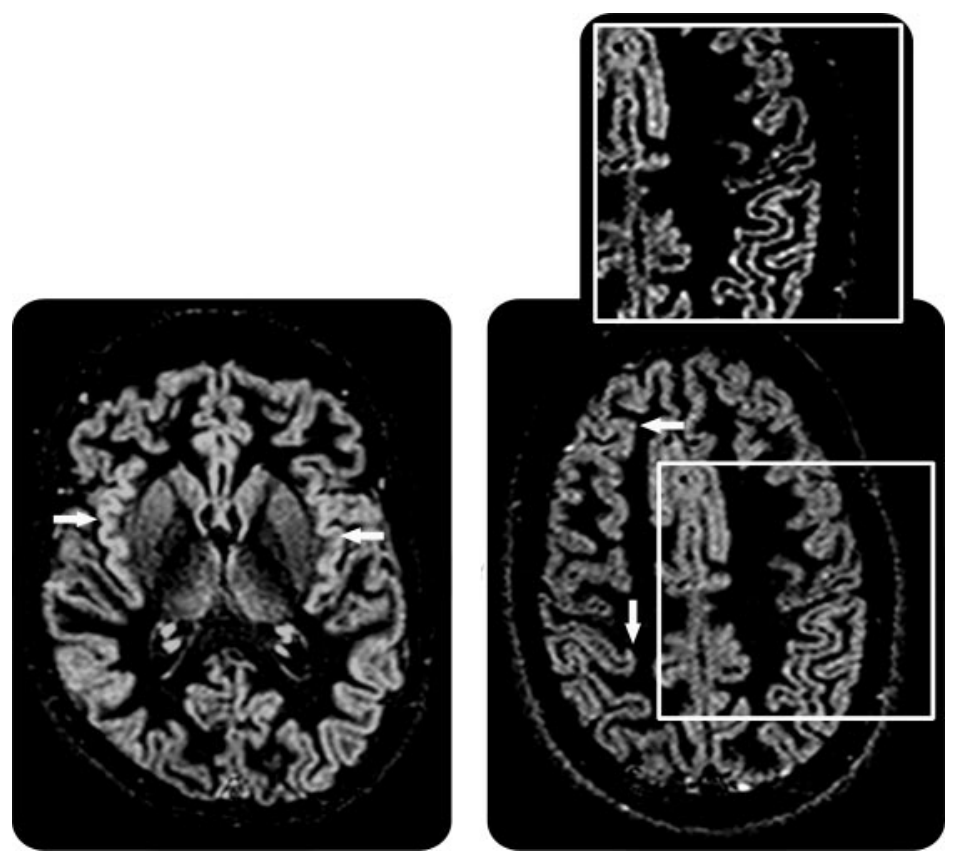

Left panel: Areas of high signal in the archicortex and paleocortex, such as the insula (arrows). Right panel: Cortical vessels may be a nuisance factor when examining DIR images; arrows indicate hyperintense vessels which may on occasion be mistaken for cortical lesions, and the box (right panel) and inset (top panel) illustrates that the cortex may appear "grainy" as a result of vessel hyperintensities.

DIR), may be used to verify suspected lesions and to distinguish them from artifacts.

Cortical lesion scoring using the recommendations. A total of 537 cortical lesions were scored by the 5 teams, with a range of 4 to 142 lesions per patient (for examples of lesions scored by only one or by multiple rating teams, see figure 3 ). Using the proposed scoring recommendations, complete agreement (i.e., all 5 teams scoring the same lesion) was reached in 104 out of 537 lesions (19.4\%), whereas 4 or more teams agreed about 212 lesions (39.5\%) and 3 or more teams out of 5 agreed on 290 lesions $(54.0 \%)$. Full agreement was generally reached more easily when lesions contained a WM part (mixed WM/GM lesions), while agreement was low in the case of smaller intracortical lesions and lesions affecting the cerebellar cortex (figure 3 ). Also, hyperintensities in the insula or any of the other archicortical and paleocortical regions (inherently higher signal intensity on DIR sequences) were rarely scored by all 5 teams (6 out of 47 lesions). The DIR sequences acquired in Padova, Amsterdam, and Basel (see table) generated the highest average agreement $(2.58,2.48$, and 2.63 teams out of 5 agreeing per lesion, respectively).

On the patient level, i.e., concerning agreement on the total number of cortical lesions per patient, the teams scored with a coefficient of variation of $42 \%$.

DISCUSSION Cortical MS lesions are difficult to visualize in vivo when using standard MRI techniques, ${ }^{5}$ and the introduction of DIR has led to a major improvement in the detection of these lesions. ${ }^{6}$ Realizing the potential of this technique, several research groups have started implementing DIR on their scanner systems and, at different sites, DIR has been used to visualize cortical lesions in patients with MS and to relate cortical lesion numbers and volumes to clinical, especially cognitive, deficits. ${ }^{9,11,21}$ Furthermore, DIR has been used to study the relation between cortical lesions and other MRI characteristics of patients with MS, such as cortical atrophy and WM damage. ${ }^{10,11}$ However, different DIR sequence parameters are currently being applied worldwide, and images are acquired at different field strengths, which makes it difficult to compare available cortical lesion data from the different groups. The aim of the consensus meeting reported here was to assess the existing DIR imaging data from different European centers, and formulate internationally useful recommendations for scoring of cortical lesions in MS. The usefulness of these recommendations was provisionally explored by determining agreement of cortical lesion scorings between different reader teams, and it was found that, when using this inherently heterogeneous set of images, more than half of the readers ( $\geq 3$ out of 5 teams) agreed on slightly more than half (54\%) of the cortical lesions scored. On a patient-by-patient level, a coefficient of variation of $42 \%$ was found. These findings indicate that it is possible to reach agreement, albeit moderate, concerning cortical lesion scoring in MS with ratings being based on available DIR data. In particular, confidence in scoring of a lesion (interteam agreement) increased in cases of large and mixed GM/WM lesions, suggesting that a comparison between available literature data on cortical lesions may become problematic if lesion characteristics depart from these features.

From a theoretical point of view, even in more homogeneous datasets than the one used for the current study, agreement on what is and what is not a cortical lesion may be challenging. Owing to 2 subsequent inversion pulses that together suppress the signal from CSF and WM, an image with a relatively low signal-to-noise is produced. ${ }^{6,12,20}$ This, in combination with a certain proneness to artifacts, such as flow and slice profile inhomogeneities (i.e., nonuniform signal in multislab imaging volumes), has already prompted researchers to replace multislab ${ }^{6}$ versions of DIR by single-slab versions. ${ }^{12,20}$ However, 

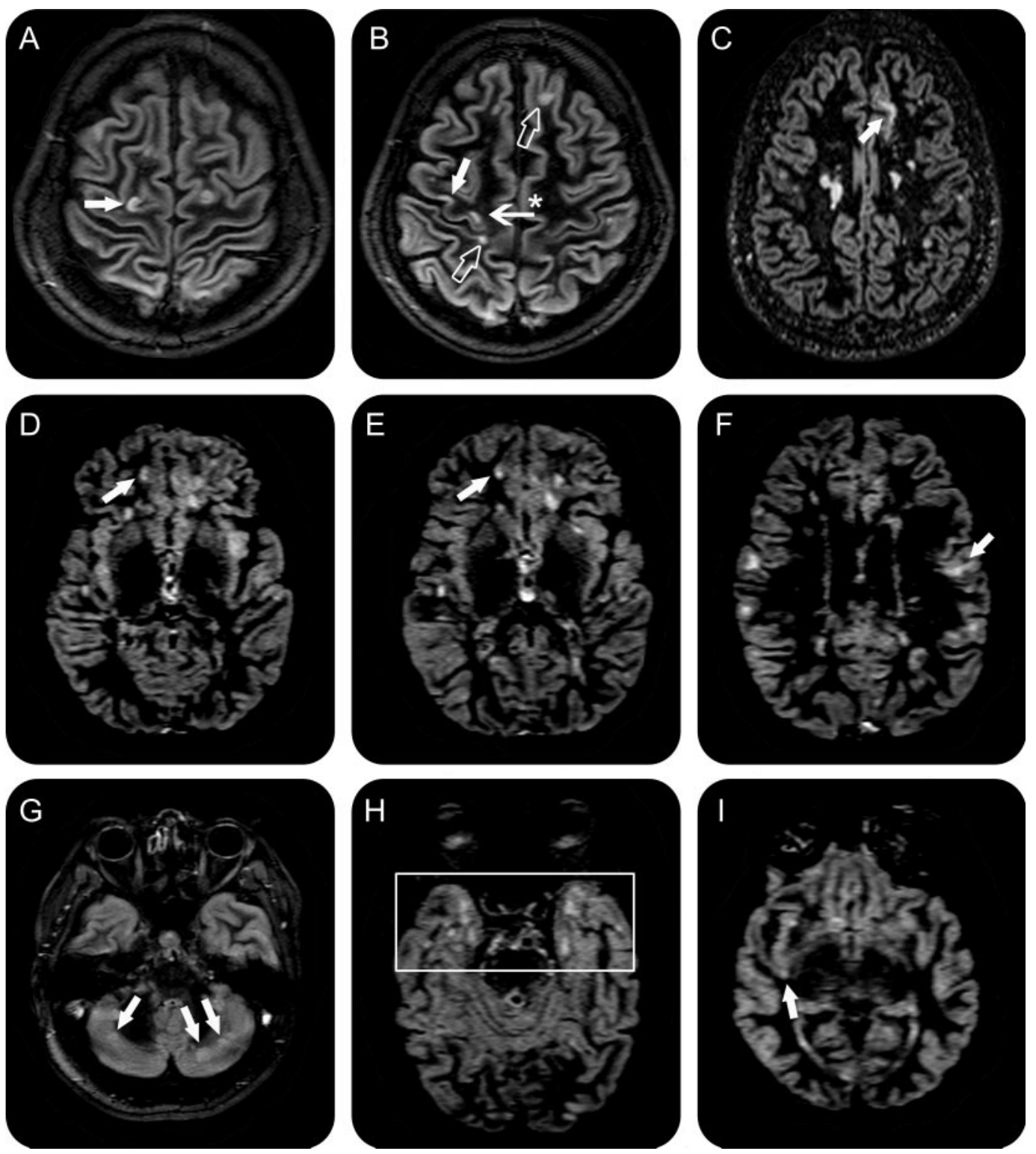

In all panels (A-I), scored intracortical lesions are indicated by closed arrows, and scored mixed white matter/gray matter lesions are indicated by open arrows. Lesions marked by all rating teams are shown in panels (A) to (C), with the exception of the juxtacortical lesion indicated by the arrow marked with an asterisk in (B), where raters disagreed about the localization of the lesion. The lesion indicated by the arrow in (D) and (E) was viewed over 2 consecutive slices to ensure its localization. Lesions scored by only 1 or 2 out of the 5 rating teams are shown in panels (G) to (I); these lesions often occur in the cerebellar gray matter (G), the medial temporal lobes $(\mathrm{H})$ (indicated by the box), and the insular cortex (I). Please see text for more details.

variation of $\mathrm{T} 1$ properties of different cortical areas, which is generally appreciated on DIR images (figure 2), may still get in the way of reliable lesion scoring, which has led to debate about whether all hyperintensities scored on DIR images are, in fact, cortical lesions. With a postmortem verification of DIR findings still lacking at this point, and given the ongoing use of DIR images to identify GM lesions, the consensus recommendations presented here sought to establish a unified set of practical rules for GM lesion scoring, applicable to currently available multicenter
DIR data. It is anticipated that, with further improved imaging techniques, and future information from postmortem work, these recommendations will be revised. However, in their present form they may provide a useful baseline against which subsequent ones can be compared.

The recommendations recommend that areas identified as GM lesions be clearly hyperintense on DIR, and cover at least 3 pixels (based on a minimal in-plane resolution of $1.0 \mathrm{~mm}^{2}$ ). These criteria were proposed to account for the relatively low signal-to- 
noise of DIR images at $1.5 \mathrm{~T}$ and $3 \mathrm{~T}$ (compared with, for example, conventional 2-dimensional T2weighted images). Furthermore, the recommendations take into account that DIR images at $1.5 \mathrm{~T}$ and $3 \mathrm{~T}$ are relatively noisy and inhomogeneous in terms of $\mathrm{T} 1$, and that cortical vessels may be a nuisance factor when scoring cortical lesions. The supportive recommendations take this further, suggesting that image readers become acquainted with signal intensities in archicortical and paleocortical areas, such as the hippocampus and insula, which may be inherently higher on DIR compared to those of neocortical areas (figure 2). Also, following suspected lesions over several slices and using other MRI contrasts like T1-weighted or phase-sensitive or fluid-attenuated inversion recovery to consider the morphologic features of observed DIR hyperintensities may further convince the reader on whether or not to score hyperintensities as cortical lesions. It should be noted that different techniques may visualize different subpopulations of cortical lesions, ${ }^{17}$ and that the absence of a signal abnormality on T1 or fluid-attenuated inversion recovery does not necessarily imply that a signal hyperintensity on DIR should be artifactual. However, one may choose to be prudent in individual cases where lesions do not match on different image contrasts. For the time being, the consortium gathered for this meeting decided against scoring subpial demyelination on DIR, as it is known from comparative MRI-histopathology studies that these lesions create extremely low contrast on MRI., ${ }^{522}$ Scoring of demyelination within the upper layers of the cortex may therefore a priori be expected to generate high numbers of false-positive results, and generally poor agreement between readers. In the future, visualization of these lesions may be facilitated by the use of higher field strengths, ${ }^{13,14}$ and hence be considered as part of updated scoring recommendations.

The final set of recommendations presented in the current article were created through discussion between MRI experts from 6 different MS centers in Europe, and were fine-tuned during image review sessions and verification of interim recommendations. The ultimate set of obligatory and supportive recommendations presented in the current article was used by the experts for the first, provisional scoring of a small selected dataset of DIR images from both patients with MS and healthy controls. Although agreement was calculated based on these ratings, the current study was not designed as a formal interrater study, and hence did not specifically aim to validate the formulated recommendations, nor to determine their sensitivity, specificity, or predictive value. Rather, these results, based on the defined scoring recommendations, should be seen as a first and tentative exploration, showing that it is possible to reach moderate consensus about roughly half of the observed cortical hyperintensities on DIR, when using currently available and heterogeneous data. Although these recommendations may enable a more integrated interpretation of DIR hyperintensities, they still leave readers divided as to the nature of almost half of the hyperintensities identified by at least one group. This suggests that increased consistency in acquisition protocols may improve future scoring agreement. The DIR sequences acquired in Padova, Amsterdam, and Basel elicited the highest average agreement per lesion, but it is difficult to recommend any of these sequences for clinical or therapeutic applications at this point, as this would require a study specifically designed for this purpose. Lesion type, size, and location, as well as field strength, image quality measures, and sequence type, are likely to have an effect on scoring agreement, even though these factors did not reach statistical significance in the current, restricted sample. The recommendations proposed here should now be studied within a more formal, prospective setting, using multicenter DIR data with similar sequence parameters and field strengths, in order to determine their practical value, and to further adapt them to improve the reliability of cortical lesion number and volume comparisons between different studies and raters in future MS studies. When sufficient reliability regarding cortical lesion assessment is reached, they might be incorporated in updated MS diagnostic criteria and an optimal DIR sequence for the clinical setting should be proposed.

\section{AUTHOR CONTRIBUTIONS}

Statistical analysis was conducted by Dr. J.J.G. Geurts and Dr. B.M.J. Uitdehaag.

\section{COINVESTIGATORS}

The current work was conducted on behalf of the MAGNIMS Study Group. The following Contributors provided patient data and discussed the contents and interpretation of the study: F. Barkhof, MD (Department of Radiology, VU University Medical Centre, Amsterdam, the Netherlands); X. Montalban, MD (Clinical Neuroimmunology Unit, Multiple Sclerosis Centre of Catalonia, Hospital Vall d'Hebron, Barcelona, Spain); N. de Stefano, MD (Department of Neurological and Behavioral Sciences, University of Siena, Siena, Italy); F. Fazekas, MD (Department of Neurology, Medical University Graz, Graz, Austria); M. Filippi, MD (Neuroimaging Research Unit, Department of Neurology, Scientific Institute and University, Ospedale San Raffaele, Milan, Italy); J. Frederiksen, MD (MS Clinic, Department of Neurology, University of Copenhagen, Glostrup Hospital, Denmark); L. Kappos, MD (Department of Neurology, University Hospital, Kantonsspital, Basel, Switzerland); D. Miller, MD (NMR Research Unit, Institute of Neurology, University College London, London, UK); J. Palace, MD (Centre for Functional Magnetic Resonance Imaging of the Brain, University of Oxford, UK); C.H. Polman, MD (Department of Neurology, VU University Medical Centre, Amsterdam, the Netherlands); M. Rovaris, MD (U.O. Sclerosi Multipla, IRCCS S. Maria Nascente, Fondazione Don Gnocchi, Milan, Italy); M. Rocca, MD (Neuroimaging Research Unit, Department of Neurology, Scientific Institute and University, Ospedale San Raffaele, 
Milan, Italy); A. Rovira, MD (Department of Radiology, Magnetic Resonance Unit, Hospital Vall d'Hebron, Barcelona, Spain); A. Thompson, MD (NMR Research Unit, Institute of Neurology, University College London, London, UK); T. Yousry, MD (NMR Research Unit, Institute of Neurology, University College London, London, UK).

\section{ACKNOWLEDGMENT}

The authors thank Dr. Hugo Vrenken and Dr. Petra J.W. Pouwels for comments.

\section{DISCLOSURE}

Dr. Geurts serves on scientific advisory boards for the Dutch MS Research Foundation and Merck Serono, and serves on the editorial board of MS International. Dr. Roosendaal receives research support from the Dutch MS Research Foundation (06-592). Dr. Calabrese reports no disclosures. Dr. Ciccarelli serves as Clinical Editor for Current Medical Literature-Multiple Sclerosis and receives support from UCLH/UCL Comprehensive Biomedical Research Centre, MS Society, and the Wellcome Trust (Advanced Fellow). Dr. Agosta serves on the speakers' bureau for Bayer Schering Pharma and has received funding for travel and speaker honoraria from Bayer Schering Pharma and Teva Pharmaceutical Industries Ltd. Dr. Chard receives research support from the Multiple Sclerosis Society Great Britain and Northern Ireland and holds stock in GlaxoSmithKline. Dr. Gass serves on the editorial board of Cerebrovascular Diseases. Dr. Huerga, Dr. Moraal, Dr. Pareto, and Dr. Rocca report no disclosures. Dr. Wattjes has received funding for travel and speaker honoraria from Bayer Schering Pharma, Janssen, and Biogen Idec. Dr. Yousry has served on scientific advisory boards for UCB and Biogen Idec; serves on the editorial board of European Radiology; and receives research support from Novartis, Biogen Idec, GlaxoSmithKline, Medical Research Council, PSP Association, Stroke Association, British Heart Foundation, and Wellcome Trust. Dr. Uitdehaag serves on a scientific advisory board for Merck Serono; served on the editorial board for Tijdschrift voor Neurologie en Neurochirurgie; receives royalties from the publication of EvidenceBased Neurology (Blackwell Publishers, 2007); serves as a consultant for Danone Research; and has received research support from the Dutch MS Research Foundation. Dr. Barkhof serves on scientific advisory boards for Lundbeck Inc., Bayer Schering Pharma, Sanofi- Aventis, UCB, Novartis, Biogen Idec, BioMS Medical, Merck Serono, and GE Healthcare; serves on the editorial boards of Brain, the Journal of Neurology, Neurosurgery, and Psychiatry, European Radiology, the Journal of Neurology, and Neuroradiology; has received speaker honoraria from Novartis, Merck Serono, and BioClinica; serves as a consultant for Sanofi-Aventis, UCB, Novartis, Biogen Idec, BioMS Medical, Medicinova, Inc., and GE Healthcare. The VUmc MS Center Amsterdam receives research support from the Dutch MS Research Foundation.

Received May 18, 2010. Accepted in final form August 30, 2010.

\section{REFERENCES}

1. Peterson JW, Bo L, Mork S, Chang A, Trapp BD. Transected neurites, apoptotic neurons, and reduced inflammation in cortical multiple sclerosis lesions. Ann Neurol 2001;50:389-400

2. Bo L, Vedeler CA, Nyland HI, Trapp BD, Mork SJ. Subpial demyelination in the cerebral cortex of multiple sclerosis patients. J Neuropathol Exp Neurol 2003;62:723-732.

3. Kutzelnigg A, Lucchinetti CF, Stadelmann C, et al. Cortical demyelination and diffuse white matter injury in multiple sclerosis. Brain 2005;128:2705-2712.

4. Geurts JJ, Barkhof F. Grey matter pathology in multiple sclerosis. Lancet Neurol 2008;7:841-851.

5. Geurts JJ, Bo L, Pouwels PJ, et al. Cortical lesions in multiple sclerosis: combined postmortem MR imaging and histopathology. AJNR Am J Neuroradiol 2005;26:572577.

6. Geurts JJ, Pouwels PJ, Uitdehaag BM, et al. Intracortical lesions in multiple sclerosis: improved detection with $3 \mathrm{D}$ double inversion-recovery MR imaging. Radiology 2005; 236:254-260.

7. Calabrese M, De Stefano N, Atzori M, et al. Detection of cortical inflammatory lesions by double inversion recovery magnetic resonance imaging in patients with multiple sclerosis. Arch Neurol 2007;64:1416-1422.

8. Simon B, Schmidt S, Lukas C, et al. Improved in vivo detection of cortical lesions in multiple sclerosis using double inversion recovery MR imaging at 3 Tesla. Eur Radiol 2010;20:1675-1683.

9. Calabrese M, Agosta F, Rinaldi F, et al. Cortical lesions and atrophy associated with cognitive impairment in relapsing-remitting multiple sclerosis. Arch Neurol 2009; 66:1144-1150.

10. Calabrese M, Rocca MA, Atzori M, et al. Cortical lesions in primary progressive multiple sclerosis: a 2-year longitudinal MR study. Neurology 2009;72:1330-1336.

11. Roosendaal SD, Moraal B, Pouwels PJ, et al. Accumulation of cortical lesions in MS: relation with cognitive impairment. Mult Scler 2009;15:708-714.

12. Pouwels PJ, Kuijer JP, Mugler JP III, Guttmann CR, Barkhof F. Human gray matter: feasibility of single-slab $3 \mathrm{D}$ double inversion-recovery high-spatial-resolution MR imaging. Radiology 2006;241:873-879.

13. Schmierer K, Parkes HG, So PW, et al. High field (9.4 Tesla) magnetic resonance imaging of cortical grey matter lesions in multiple sclerosis. Brain 2010;133:858-867.

14. Mainero $C$, Benner $T$, Radding A, et al. In vivo imaging of cortical pathology in multiple sclerosis using ultra-high field MRI. Neurology 2009;73:941-948.

15. Wattjes MP, Barkhof F. High field MRI in the diagnosis of multiple sclerosis: high field-high yield? Neuroradiol 2009;51:279-292.

16. Nelson F, Poonawalla AH, Hou P, et al. Improved identification of intracortical lesions in multiple sclerosis with phase-sensitive inversion recovery in combination with fast double inversion recovery MR imaging. AJNR Am J Neuroradiol 2007;28:1645-1649.

17. Bagnato F, Butman JA, Gupta $S$, et al. In vivo detection of cortical plaques by MR imaging in patients with multiple sclerosis. AJNR Am J Neuroradiol 2006;27:2161-2167.

18. Nelson F, Poonawalla A, Hou P, Wolinsky JS, Narayana PA. 3D MPRAGE improves classification of cortical lesions in multiple sclerosis. Mult Scler 2008;14:12141219.

19. Bagnato F, Yao B, Cantor F, et al. Multisequence-imaging protocols to detect cortical lesions of patients with multiple sclerosis: observations from a post-mortem 3 Tesla imaging study. J Neurol Sci 2009;282:80-85.

20. Moraal B, Roosendaal SD, Pouwels PJ, et al. Multicontrast, isotropic, single-slab 3D MR imaging in multiple sclerosis. Eur Radiol 2008;18:2311-2320.

21. Calabrese M, Rocca MA, Atzori M, et al. A 3-year magnetic resonance imaging study of cortical lesions in relapseonset multiple sclerosis. Ann Neurol 2010;67:376-383.

22. Geurts JJ, Blezer EL, Vrenken H, et al. Does high-field MR imaging improve cortical lesion detection in multiple sclerosis? J Neurol 2008;255:183-191. 


\section{Neurology}

\section{Consensus recommendations for MS cortical lesion scoring using double inversion recovery MRI}

J.J.G. Geurts, S.D. Roosendaal, M. Calabrese, et al.

Neurology 2011;76;418-424 Published Online before print January 5, 2011

DOI 10.1212/WNL.0b013e31820a0cc4

\section{This information is current as of January 5, 2011}

\section{Updated Information \&} Services

References

Citations

Subspecialty Collections

Permissions \& Licensing

Reprints including high resolution figures, can be found at: http://n.neurology.org/content/76/5/418.full

This article cites 22 articles, 5 of which you can access for free at: http://n.neurology.org/content/76/5/418.full\#ref-list-1

This article has been cited by 25 HighWire-hosted articles: http://n.neurology.org/content/76/5/418.full\#\#otherarticles

This article, along with others on similar topics, appears in the following collection(s):

All Clinical Neurology

http://n.neurology.org/cgi/collection/all_clinical_neurology

All Demyelinating disease (CNS)

http://n.neurology.org/cgi/collection/all_demyelinating_disease_cns

Memory

http://n.neurology.org/cgi/collection/memory

MRI

http://n.neurology.org/cgi/collection/mri

Multiple sclerosis

http://n.neurology.org/cgi/collection/multiple_sclerosis

Information about reproducing this article in parts (figures,tables) or in its entirety can be found online at:

http://www.neurology.org/about/about_the_journal\#permissions

Information about ordering reprints can be found online:

http://n.neurology.org/subscribers/advertise

Neurology ${ }^{\circledR}$ is the official journal of the American Academy of Neurology. Published continuously since 1951, it is now a weekly with 48 issues per year. Copyright Copyright (? 2011 by AAN Enterprises, Inc.. All rights reserved. Print ISSN: 0028-3878. Online ISSN: 1526-632X.

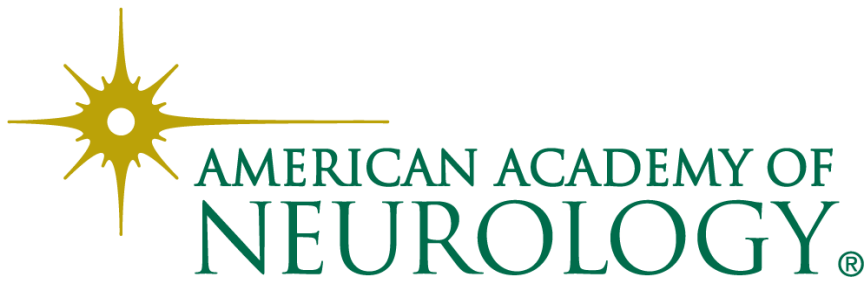

